\title{
Improving the Performance Metric of Wireless Sensor Networks with Clustering Markov Chain Model and Multilevel Fusion
}

\author{
Saeed Havedanloo ${ }^{1}$ and Hamid Reza Karimi ${ }^{2}$ \\ ${ }^{1}$ Departmant of ICT, Faculty of Engineering and Science, University of Agder, 4898 Grimstad, Norway \\ ${ }^{2}$ Department of Engineering, Faculty of Engineering and Science, University of Agder, 4898 Grimstad, Norway
}

Correspondence should be addressed to Hamid Reza Karimi; hamid.r.karimi@uia.no

Received 17 October 2012; Accepted 11 November 2012

Academic Editor: Ligang Wu

Copyright (c) 2013 S. Havedanloo and H. R. Karimi. This is an open access article distributed under the Creative Commons Attribution License, which permits unrestricted use, distribution, and reproduction in any medium, provided the original work is properly cited.

\begin{abstract}
The paper proposes a performance metric evaluation for a distributed detection wireless sensor network with respect to IEEE 802.15.4 standard. A distributed detection scheme is considered with presence of the fusion node and organized sensors into the clustering and non-clustering networks. Sensors are distributed in clusters uniformly and nonuniformly and network has multilevel fusion centers. Fusion centers act as heads of clusters for decision making based on majority-like received signal strength (RSS) with comparison the optimized value of the common threshold. IEEE 802.15.4 Markov chain model derived the performance metric of proposed network architecture with MAC, PHY cross-layer parameters, and Channel State Information (CSI) specifications while it is including Path-loss, Modulation, Channel coding and Rayleigh fading. Simulation results represent significant enhancement on performance of network in terms of reliability, packet failure, average delay, power consumption, and throughput.
\end{abstract}

\section{Introduction}

In the recent years, employments of wireless sensor networks (WSNs) have increased in many aspects of modern lifestyle. Those applications have motivated the researchers around the world to attempt into this field and investigate Quality of Service (QoS) and improve performance and efficiency of network. Usually, wireless sensor networks are supposed to be in harsh environments; consequently, performance metric evaluation at the real situation is difficult, where human intervention for evaluating process, even maintenance, repair, or fix purposes are in jeopardy. Hence, performance evaluation based on the mathematical model of network and simulation is highly considered. Sometimes controlling a process in the large scale needs sensing a unique phenomenon of interest with several sensors. An actuator reacts precisely in relation to decision which is made based on received signals from sensors. Fusion of multiple sensing signals makes a decision more accurate than just one sensor and consequently increases system efficiency.
To address problem, a novel performance evaluation framework would be proposed. Mathematical model framework of a decentralized distributed detection is studied in cluster-based network with a Markov chain model for IEEE 802.15.4 Medium Access Control (MAC) with respect to CSMA/CA mechanism interplay by physical layer and channel state information. The framework investigates appropriated strategies by configuration of wireless sensor nodes based on the optimal tuning of IEEE 802.15.4 MAC and PHY layer key parameters [1]. Head node of each cluster is called Fusion Center (FC). Decision making at fusion node performs with respect to majority-like reception of RSS with Maximum-Likelihood Test.

Performance metric is evaluated for a clustering network topology with respect to a Markov chain model for CSMA/CA medium access control which proposed in [2] for a single node. Model describes a generalized analytical of the slotted CSMA/CA mechanism of beacon-enabled IEEE 802.15.4 with retry limits for each packet transmission. Behavior of the Markov model proposed at [2] is 
describing CSMA/CA algorithm for a single node within star network with $N$ sensor nodes whereas our attempt updates performance metric equations with clustering topology and is accompanied by FCs. Model in [2] is only considered to packet collision probability as case of loss. Nevertheless, physical layer and channel state are provoking factors to loss indeed [3]. Therefore, physical-layer and CSI specification such as modulation and channel coding are utilized through the equations as a probability that denotes with $\left(P_{c s i}\right)$. Network is supposed to be high data rate generation for assessment of performance. Simulation is carried out to represent probability of decision error at FC $\left(P_{e}\right)$ in a clustered network with significant enhancement on performance metric in terms of reliability $\left(R_{c}\right)$, packet failure $\left(P_{f}\right)$, average delay $\left(\mathbb{E}_{c}^{\text {(av) }}\right)$, power consumption with considering different operation modes, idle $\left(P_{i}\right)$, sensing $\left(P_{\mathrm{sc}}\right)$, transmission $\left(P_{t}\right)$ and receiving $\left(P_{r}\right)$, and also Network aggregation throughput $\left(S_{c}\right)$.

\section{Related Work}

In the literature, for instance see [4] and the references therein, wireless sensor network is studied with a small amount of sensors and low signal to noise ratio (SNR), distributed detection, and decision making fusion rules carried out on multi-bit knowledge of local detecting sensors with Monte-Carlo simulation methods. The performance of proposed decision fusion rules is integrated with parameters such as channel Rayleigh fading and adaptive Gaussian noise. In $[5,6]$, the authors with respect to similar field of efforts in [7], proposed a simulation-based analysis impact of data fusion mechanisms in a Zigbee sensor network. It is used to monitor a particular constant binary phenomenon and evaluated performance indicators of interest, for example, Bit Error Rate (BER) and networking oriented (delay and aggregate throughput). In $[8,9]$ a distributed detection (DD) system is considered for multiple sensors/detectors work, collaboratively and the fusion center is responsible for the final decision-making task based on information gathered from local sensors; moreover, the integration of wireless channel conditions in algorithm design is also taken into the account (also see $[10,11])$. In [12], an important channel dynamic is well defined; their studies are represented by the behavior of a real link impact in low-power wireless networks. In particular, there is a large transitional region in wireless link quality which is characterized by significant levels of unreliability and asymmetry, significantly impacting on performance of higher-layer protocols. In [3], the authors used the first way to better understand IEEE 802.15.4 standard. Indeed, they provided a comprehensive model, able more faithfully to mimic the functionalities of this standard at the PHY and MAC layers. They have proposed a combination of two relevant models for the two layers. The PHY layer behavior is reproduced by a mathematical framework, which is based on radio and channel models, in order to quantify link reliability. In $[2,13,14]$ the authors proposed a generalized analysis of the IEEE 802.15.4 medium access control (MAC) protocol with focus on CSMA/CA algorithm in terms of reliability, delay, and energy consumption (for more see $[15,16])$. The rest of this paper is as follows. In Section 3, we will describe the analytical framework to evaluate performance metric. This section consists of the several subsections. In Section 4 we will represent simulation results and finally Section 5 would conclud the paper.

\section{Problem Framework}

In this section, we investigate the problem of decentralized distribution detection particularly when the sensor nodes detect a constant binary phenomenon. Sensing data packages and forwards to access point (AP) through intermediate fusion center (FC). Decision making fusion rule performs at FC with majority-like signal power level reception compared to an optimized threshold. Two ideal and noisy (nonideal) channels assume and channel state information (CSI) considers with its impacts on decision-making fusion rule Probability of decision error measures at FC versus signal to noise ratio with modulation and channel coding influences. Sensor nodes distribution at each cluster is supposed to be uniform and nonuniform.

The rest of section is organized as Sections 3.1 and 3.2 depict sensing model and distributed detection in Parallel Fusion Architecture, respectively and Section 3.3 describes distributed detection in clustered Sensor Networks. Section 3.4 comprises communication channel state information such as The Rayleigh fading, path-loss and modulation, and channel coding. Section 3.5 describes medium access control role on clustered network and its performance metric equations with presence FC and impacts of CSI.

3.1. The Sensing Model. According to the stochastic geometry of sensing model, distribution of the nodes over the observing region $\mathrm{A}$ can be modelled by a homogeneous Poisson point process (PPP) with intensity $\rho$. Sensing model is a isotropic signal source model for detecting phenomena of interest (PoI) with path loss factor $\alpha$ depends on distance of sensor from PoI and type of signal (chemical contamination, sound, radioactive radiation, etc.) [17]. Here, we assume $\alpha$ is equal to 1 and sensor distance from PoI is $d=1$ meter. Due to sensors are integrated with transmitters as a element of a WSN, thus, the received detection signal strength to sensor with a distance $d$ away from the PoI is given by:

$$
\begin{gathered}
S(d)=\frac{S_{0}}{d^{\alpha}}, \\
\mathbb{P}\left\{N_{t}=n_{t}\right\}=\frac{\lambda_{t}^{n_{t}} \exp \left(-\lambda_{t}\right)}{n_{t} !}, \quad n_{t} \geq 0,
\end{gathered}
$$

where $N_{t}$ is a Poisson r.v. with mean $\lambda_{t}=\mathbb{E}\left\{N_{t}\right\}=\rho|\mathrm{A}|$, whereas $\rho$ is intensity of distribution nodes over observing a finite region of phenomenon with size $|\mathrm{A}|$. We suppose the nodes sensing periodically independent condition whether $\mathrm{PoI}$ is absent or present. Particularly, while the $\mathrm{PoI}$ is present, observations are not similar between nodes belong into the 


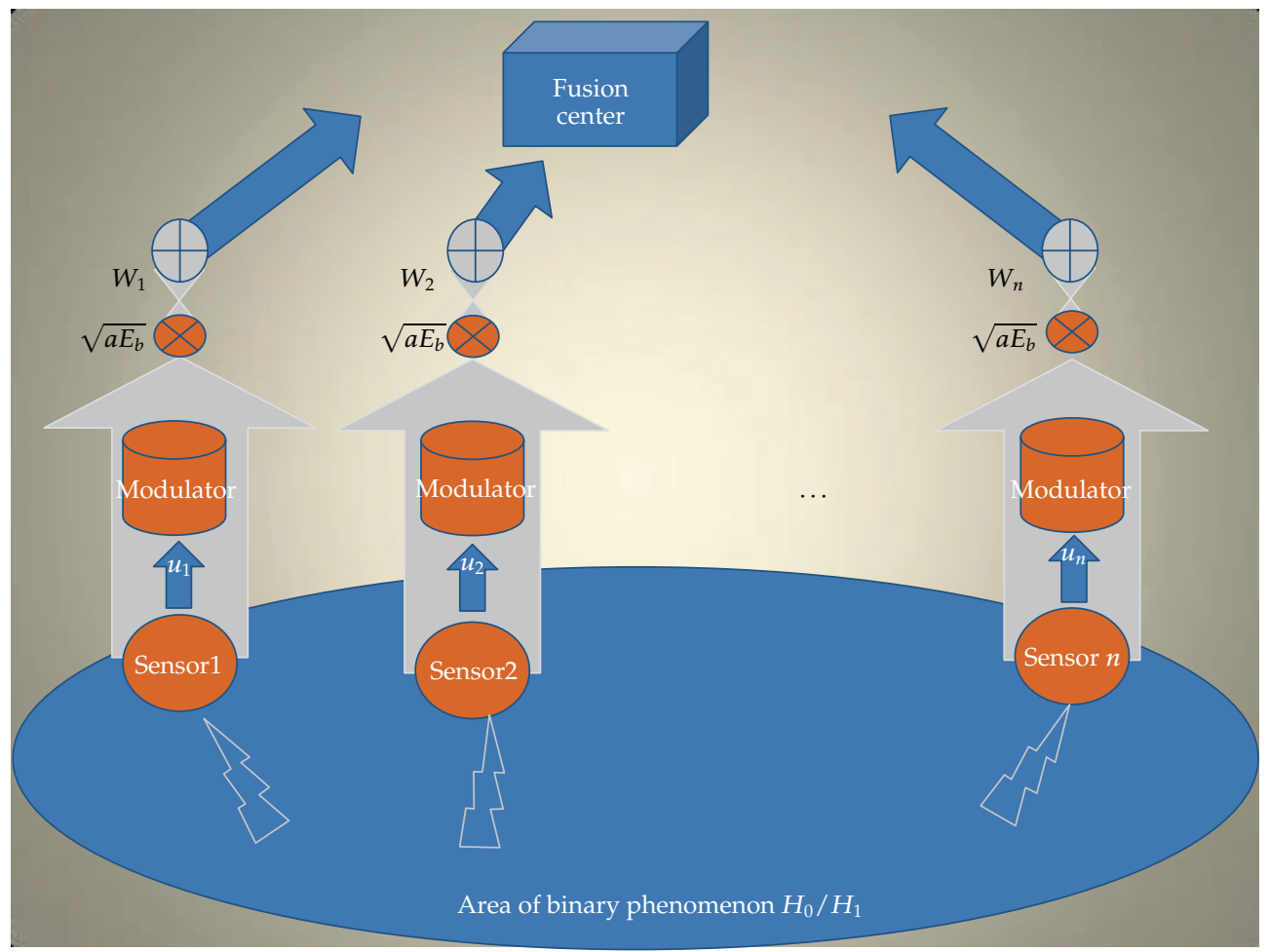

FIGURE 1: Parallel fusion architecture.

same group of sensors. In this case, observation independently remarks at each sensor node after proper sampling and processing is given by

$$
y_{n}= \begin{cases}z_{n}, & \text { when PoI is absent, } \\ \sqrt{S\left(d_{n}\right)}+z_{n}, & \text { when PoI is present }\end{cases}
$$

where $n=1,2, \ldots, N_{t}, z_{n}$ is an independent observation Gaussian distribution noise with zero-mean and variance $\sigma_{z}^{2}$. $S\left(d_{n}\right)$ is the received signal strength at the $n$th node with a distance $d_{n}$ far from the PoI given by (1). Thus, problem status could be defined as follows:

$$
H= \begin{cases}H_{0}: & \text { absent PoI with probability } p_{0}, \\ H_{1}: & \text { present PoI with probabilty } 1-p_{0} .\end{cases}
$$

Information is gathered from observers of PoI, located in center region $A$ (environment of observed PoI); hence, equal probability is assumed in term of present or absent PoI, where $p_{0}=\mathbb{P}\left\{H=H_{0}\right\}, \mathbb{P}\{\cdot\}$ being the probability of a given PoI.

\subsection{Distributed Detection in Parallel Fusion Architecture.} Sensor nodes are organized within Parallel Fusion Architecture (PFA) which is represented on Figure 1. Each sensor independently detects the event under observation and generates information and sends to FC through an ideal communication link. Information could be sequence of bits as symbol of present or absent PoI. According to (2) and (3), sensors send 1 bit unit information to FC for decision making. A basic equation derived for received sensor observation signal at the FC from the $n$th sensor node is given by:

$$
r_{n}=c_{E}+w_{n}
$$

where $c_{E}=\sqrt{a E_{b}} u_{n}$ and $w_{n}$ is a channel noise modeled zero-mean Gaussian distribution with variance $N_{0} / 2$ and across the nodes there is independent identical distribution (i.i.d). $E_{b}$ is transmission energy per bit and $a$ is up-link path loss coefficient between sensor node and FC. Assume $a$ is identical for all nodes. The $u_{n}$ is quantized local decision for observation of an event and characterized with two levels of unit function as follow:

$$
u_{n}= \begin{cases}+1: & \text { when } \widetilde{H}\left(y_{n}\right)=H_{1}, \\ -1: & \text { when } \widetilde{H}\left(y_{n}\right)=H_{0}\end{cases}
$$

whereas $\widetilde{H}\left(y_{n}\right)$ is the decision that made at the $n$th node [17]. The FC would be synchronized with whole nodes in the region A because of FC sends a beacon periodically when we want to retrieve observation data. All nodes exactly trigger and send observing data to corresponding fusion node at region $\mathrm{A}$. With hypothesis ideal communication channels, decision is made at FC with Likelihood Raito Test (LRT) level of received signal by comparison an optimized common threshold value which denotes by $\xi$. Threshold level could be adapted and trained during detection period according to level of transmission signal power. 


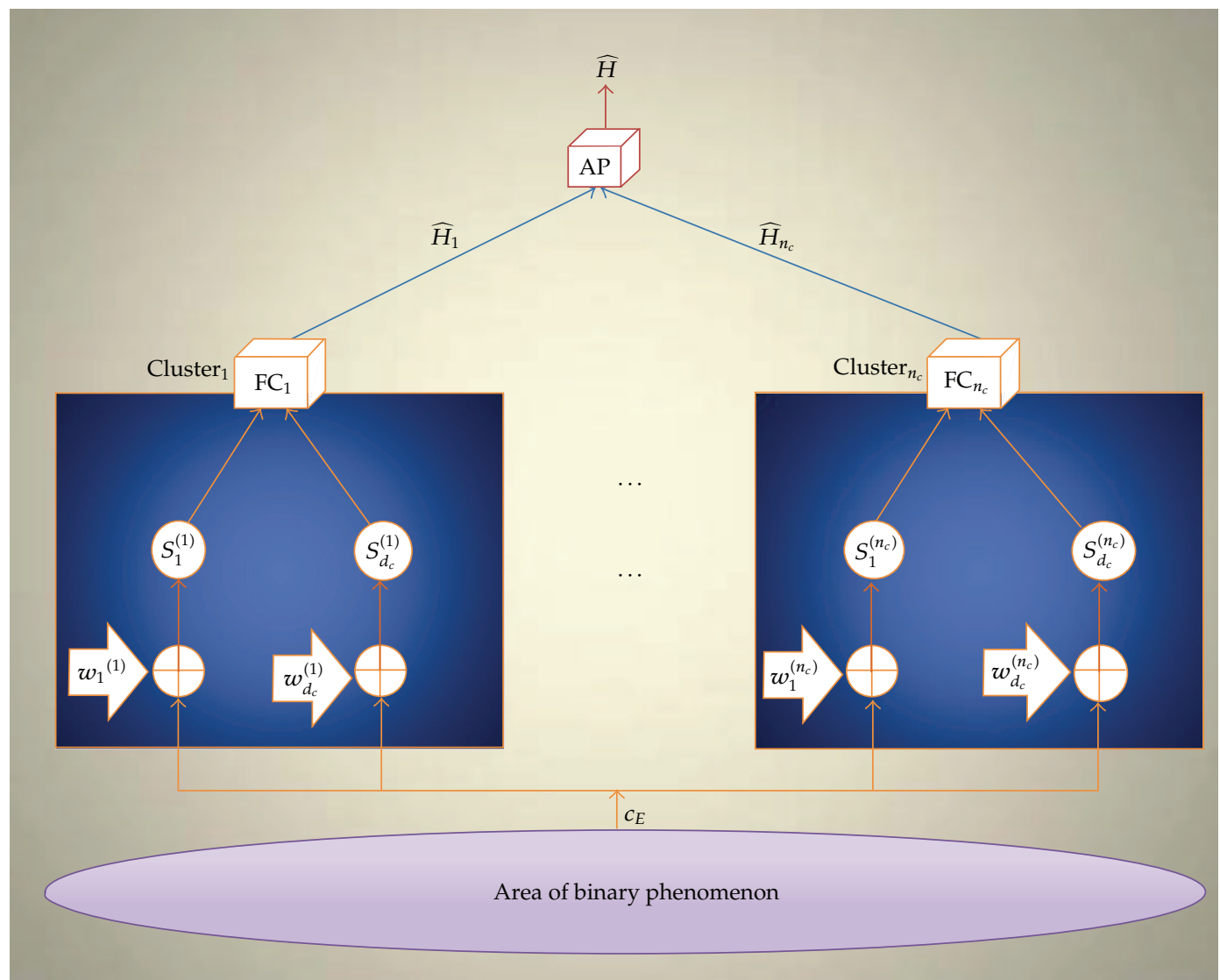

FIGURE 2: Block diagram of a clustered sensors network.

3.2.1. LRT with Neyman-Pearson Hypothesis Testing. Here, observing signal received to fusion node might be affected by many factors in an unforeseen manner, hence, the decisionmaking would be doing necessarily statistical. This formulates with a decision rule based on optimality criterion. Normally, optimal criteria are using three major methods, the Bayes risk criterion, the min-max criterion, and the Neyman-Pearson (NP) criterion. LRT is performed regarding NP criterion. Under NP criterion, the optimal decision rule derives from an LRT choosen based on the null and alternative hypotheses conditional probabilities:

$$
\frac{\mathbb{P}\left\{\mathbf{r} \mid H_{1}\right\}}{\mathbb{P}\left\{\mathbf{r} \mid H_{0}\right\}} \underset{H_{0}}{\stackrel{H_{1}}{>}} \xi,
$$

whereas data vector $\mathbf{r}$ is given under the alternative as $\mathbb{P}\left\{\mathbf{r} \mid H_{1}\right\}$ and data vector $\mathbf{r}$ under the null hypothesis as $\mathbb{P}\left\{\mathbf{r} \mid H_{0}\right\}$. FC decision performs based on the $N_{t}$ received observations of nodes. The vector $\mathbf{r}$ denotes as a gain of received signal in ideal Binary Symmetric Channels (BSCs). This is corresponding to $N_{t}$ specified in (5). Nevertheless, the $\xi$ for simplicity is adapted with $\sqrt{\mathrm{SNR}} / 2$ where SNR $=$ $a E_{b} / N_{0}$ is received signal energy per bit per noise power spectral density, can be expressed using signal to noise (SNR), to FC from each sensor node through communication channel. The received signals vector from $N_{t}$ sensor nodes is considered as follow:

$$
\mathbf{r}=\left[r_{1}, \ldots, r_{i}\right]^{T}, \quad i=\left(1, \ldots, N_{t}\right) .
$$

With the Bayesian approach, a priori probabilities of the absent or present hypothesis PoI are $\mathbb{P}\left\{H_{0}\right\}$ and $\mathbb{P}\left\{H_{1}\right\}$ at fusion center, respectively. Probability of decision error is defined at fusion center as follow:

$$
P_{e}=\mathbb{P}\left\{\widehat{H}=H_{1} \mid H_{0}\right\} \mathbb{P}\left\{H_{0}\right\}+\mathbb{P}\left\{\widehat{H}=H_{0} \mid H_{1}\right\} \mathbb{P}\left\{H_{1}\right\} .
$$

3.3. Distributed Detection in Clustered Sensor Networks. A network with $n$ sensors observes a common binary phenomenon whose status is defined at (4) with $p_{0}=\mathbb{P}\{H=$ $\left.H_{0}\right\}, \mathbb{P}\{\cdot\}$ denotes the probability of given PoI. The $n$ sensors might be organized into several clusters whereas number of cluster is $n_{c}<n$ sensor nodes. Sensors belong to a cluster working as a RFD (Reduce Function Device) just communicates with corresponding FC which is a FFD (Full Function Device). Each cluster with collection of sensors is a PFA represented in Section 3.2 and Figure 2 shows $n_{c}$ cluster-based architecture [6]. The sensors are distributed in each cluster uniformly or nonuniformly. Initially, the channel between the sensors and fusion center is supposed to be an ideal communication link such as a Binary Symmetric 
Channels (BSCs) with probability $p$ cross-over, memoryless communication. To continue, wireless channel also would be a non-ideal with respect to CSI specification.

3.3.1. Data-Fusion Model. Decision is made at fusion node and carries out with majority-like mechanism. In some literature this method is called consensus flooding or voting mechanism. Basically, this mechanism is based on majority similar received signal from sensors on the same cluster and event under observe in precise time. According to Figure 2 two-level fusion is shown; in first level, each cluster contains $d_{c}$ distributed sensors uniformly and $n_{c}$ is number of clusters, thus, $n=d_{c} \times n_{c}$ is number of all sensors in network. $k=\left[d_{c} / 2\right]+1$ is acceptable floor of majority-like for first level of fusion. In second level, decision-making is performed at access Point (AP) similarly with assuming FCs as $n_{c}$ sensors. Obviously, AP accepts mechanism with at least $k_{f}=$ $\left[n_{c} / 2\right]+1$ majority-likes. Non-uniform distribution of sensors is defined as unequal number of sensors for each cluster. It denotes clusters size vector by $D \triangleq\left\{d_{c}^{(1)}, d_{c}^{(2)}, \ldots, d_{c}^{\left(n_{c}\right)}\right\}$, where $d_{c}^{(i)}$ is the number sensors in the ith cluster $(i=1,2$, $\left.\ldots, n_{c}\right)$ and $\sum_{i=1}^{n_{c}} d_{c}^{(i)}=N$. The probability of decision error in a generic scenario with non-uniform clustering can be evaluated as below:

$$
\begin{array}{r}
P_{e}=p_{0} \sum_{i=k_{f}}^{n_{c}} \sum_{j=1}^{\left(\begin{array}{c}
n_{c} \\
i
\end{array}\right)} \prod_{\ell=1}^{n_{c}}\left\{c_{i, j}(\ell) p_{\ell}^{1 \mid 0}+\left(1-c_{i, j}(\ell)\right)\left(1-p_{\ell}^{1 \mid 0}\right)\right\} \\
+\left(1-p_{0}\right) \sum_{i=0}^{k_{f}-1} \sum_{j=1}^{\left(\begin{array}{c}
n \\
i
\end{array}\right)} \prod_{\ell=1}^{n_{c}}\left\{c_{i, j}(\ell) p_{\ell}^{1 \mid 1}\right. \\
\left.+\left(1-c_{i, j}(\ell)\right)\left(1-p_{\ell}^{1 \mid 1}\right)\right\},
\end{array}
$$

where $p_{\ell}^{1 \mid 1} \triangleq\left\{p_{1}^{1 \mid 1}, p_{2}^{1 \mid 1}, \ldots, p_{n_{c}}^{1 \mid 1}\right\}$ represents probability of success and $p_{\ell}^{1 \mid 0} \triangleq\left\{p_{1}^{1 \mid 0}, p_{2}^{1 \mid 0}, \ldots, p_{n_{c}}^{1 \mid 0}\right\}$ represents probability of failure decides at FC [9]. $\mathbf{c}_{i, j}=\left(c_{i, j}(1), \ldots, c_{i, j}\left(n_{c}\right)\right)$ is a vector which designates the $j$ th configuration of the decisions from the first-level FCs in a case with $i, 1 s$ and $n_{c}-i, 0 s[5,12]$. On the other words, $c_{i, j}$ can be represented by $\operatorname{string}(i, j, \ell)=$ 1 if there is a success, corresponding to a decision, at $\ell$ th FC or AP, in favor of $H_{1}$, whereas it is 0 if there is a failure, corresponding to a decision, at $\ell$ FC or AP in favor of $H_{0}$. $\operatorname{string}(i, j, \ell)$ could be an auxiliary binary function used to distinguish, in the repeated trials formula, between a success and a failure $[12,18]$. For example, possible configuration for $n_{c}=4$ clusters is illustrated in Table 1 .

3.4. Communication Channel State Information. In this section, channel rules will be explained in interplaying with decision-making at fusion. Generated packet bits from detected event sequentially, bit to bit would be sent to fusion node through a communication channel. The impact of channel condition or channel state information (CSI) is significant on decision which would be made at fusion node. In addition to sensor observation quality, probability
TABLE 1: Possible configuration of $\mathbf{c}_{i, j}$ for $n_{c}=4$ clusters.

\begin{tabular}{llc}
\hline$i$ & $j$ & $c_{i, j}$ \\
\hline 0 & 1 & 0000 \\
\hline & 1 & 1000 \\
& 2 & 0100 \\
& 3 & 0010 \\
& 4 & 0001 \\
\hline & 1 & 1100 \\
& 2 & 1010 \\
& 3 & 1001 \\
& 4 & 0101 \\
& 5 & 0110 \\
& 6 & 0011 \\
\hline 4 & 1 & 1110 \\
& 2 & 1011 \\
& 3 & 0111 \\
& 4 & 1101 \\
\hline
\end{tabular}

of decision error $\left(P_{e}\right)$ at FC completely is related to channel condition and Received Signal Strength Indication (RSSI). Therefore, new element is taken into the account as CSI probability of channel which is denoted by $P_{\mathrm{csi}}$. Impact of $P_{\mathrm{csi}}$ will investigate decision-making accuracy. Here, the sensor network is modeled with no interference impact (orthogonal transmission) because of an exact scheduling between the sensors and fusion node or AP. A beacon message transmits periodically for synchronization to each sensor node when FC and AP are ready for PoI sample reception.

3.4.1. The Rayleigh Fading. Equation (5) with Rayleigh fading is given by:

$$
r_{i}=f_{i}\left(2 c_{i}-1\right) \sqrt{E_{c}}+w_{i}, \quad i=1, \ldots, N+L,
$$

where $f_{i}$ is a random variable with Rayleigh distribution which is perfectly coherent demodulation and $c_{i} \in\{0,1\}$ is the symbol transmitted from a sensor, $c_{i}$ is an information bit from sensor nodes [9]. The total number of transmission in sensor network is $N+L$ whereas, $N$ is number of sensors and $L$ is bits according to the parity-check equations of the Hamming code. The $E_{c}$ is the energy per coded bit whereas $E_{c} \triangleq R_{c} E_{b} . E_{b}$ denotes the energy per bit information and $R_{c}=1 / M$ being code rate that interpreted as a system embedding a repetition code at each sensor when $M$ is consecutive and independent observations of the same phenomenon for a sensor network with multiple observations [18]. A systematic block channel code hypothesizing that each sensor makes a single observation, by using Hamming systematic block code, generates parity bits and sends them to the FC or AP. For $N=k=4$ observer sensors generate $L=n-k=3$ bits according to the parity-check equations. It remarks $(n, k)=(7,4)$ systematic Hamming code [8]. 
The total number of transmission acts in the proposed sensor network is $N+L . R_{c}$ is computed in this distributed coded scheme $R_{c}=N /(N+L)=4 / 7$. Bit Error Rate (BER) with QPSK modulation at fusion node for Rayleigh fading channel is given by:

$$
p^{\text {Rayleigh }}=\frac{1}{2}\left[1-\sqrt{\frac{R_{c} \gamma_{b}}{1+R_{c} \gamma_{b}}}\right]
$$

where $\gamma_{b} \triangleq E_{b} / N_{0}$ is SNR received at Fusion node or AP [9].

3.4.2. Pathloss. According to channel model distance $(d)$ between transmitter and receiver (FC or AP), the received power $P_{r}$ in $\mathrm{dB}$ is as follow:

$$
\begin{gathered}
P_{r}(d)=P_{t}-P L\left(d_{0}\right)-10 \eta \log _{10}\left(\frac{d}{d_{0}}\right)+N(0, \sigma), \\
P L\left(d_{0}\right)=20 * \log _{10}(f),
\end{gathered}
$$

where $P_{t}$ is the output power, $\eta$ is the pathloss exponent which takes the rate of signal attenuation based on different environment obtains with empirical measurement [12]. $N(0, \sigma)$ is a Gaussian random variable with mean 0 and variance $\sigma$ (standard deviation due to multipath shadowing effects). $\operatorname{PL}\left(d_{0}\right)$ is power attenuation at source with distance $d_{0}$ with frequency $f=v / \lambda, v$ is velocity light and $\lambda$ is wavelength. Equation (13) is an isotropic transmission. $\mathrm{SNR}$ in $\mathrm{dB}\left(\gamma_{\mathrm{dB}}\right)$ as a function of distance (meter) is:

$$
\gamma(d)=P_{r}(d)-P_{n}
$$

where $P_{n}$ is noise floor, more details see [12]. With substitute consequently,

$$
\gamma_{\mathrm{dB}}(d)=P_{t}-P L\left(d_{0}\right)-10 \eta \log _{10}\left(\frac{d}{d_{0}}\right)-N(0, \sigma)-P_{n} .
$$

3.4.3. Modulation and Channel Coding. The QPSK Modulation and NRZ (non-return zero) channel coding impact, respectively, are:

$$
p_{b}=Q\left(\sqrt{2 \gamma(d) \frac{B_{N}}{R}}\right)
$$

where $\gamma_{d}=10^{\gamma_{\mathrm{dB}} / 10}$ and $B_{N}$ is noise bandwidth and $R$ is bit data rate with channel coding given by,

$$
P_{\mathrm{csi}}=\left(1-p_{b}\right)^{8 e}\left(1-p_{b}\right)^{8(b-e)}
$$

where $e$ is Preamble length, $b$ is frame length, for more details see [12]. Rewriting (12) with channel state probability for QPSK modulation and NRZ channel coding we get

$$
P_{\mathrm{csi}}^{\text {Rayleigh }}=\frac{1}{2}\left[1-\sqrt{\frac{R_{c} \gamma_{b}(d)}{1+R_{c} \gamma_{b}(d)}}\right] \text {. }
$$

Probability of decision error $P_{e}$ at Fusion or AP given in $[6,9]$ and updated with $P_{\mathrm{csi}}^{\text {Rayleigh }}$ is

$$
\begin{array}{r}
P_{e}=p_{0} \sum_{i=k_{f}}^{n_{c}} \sum_{j=1}^{\left(\begin{array}{c}
n \\
i
\end{array}\right)} \prod_{\ell=1}^{n_{c}}\left\{c_{i, j}(\ell) q^{1 \mid 0}+\left(1-c_{i, j}(\ell)\right)\left(1-q^{1 \mid 0}\right)\right\} \\
+\left(1-p_{0}\right) \sum_{i=0}^{k_{f}-1} \sum_{j=1}^{\left(\begin{array}{c}
n \\
i
\end{array}\right)} \prod_{\ell=1}^{n_{c}}\left\{c_{i, j}(\ell) q^{1 \mid 1}\right. \\
\left.+\left(1-c_{i, j}(\ell)\right)\left(1-q^{1 \mid 1}\right)\right\},
\end{array}
$$

where $q^{1 \mid 0}=p_{\ell, \mathrm{csi}}^{1 \mid 0, \text { Rayleigh }}, q^{1 \mid 1}=p_{\ell, \mathrm{csi}}^{1 \mid 1, \text { Rayleigh }}$, and $\ell=$ $\left\{1, \ldots, n_{c}\right\}$.

3.5. Medium Access Control Role on Clustered Network. Basically, Markov chain and performance metric expression proposed in $[2,14]$ are considered with fusion and clustered network (also see [19]). Three major parameters which reformed into scenario are the probability of a node attempts a first carrier sensing (CCA1) in randomly chosen time slot is denoted with $t$ and given by

$$
\tau=\left(\frac{1-x^{m+1}}{1-x}\right)\left(\frac{1-y^{n+1}}{1-y}\right) \tilde{b}_{0,0,0},
$$

where approximation of state probability is

$$
\begin{aligned}
\tilde{b}_{0,0,0} \approx & \frac{W_{0}}{2}(1+2 x)(1+y)+L_{s}\left(1-x^{2}\right)(1+y) \\
& +K_{0}\left(\left(P_{c}\left(1-x^{2}\right)\right)^{2}\left(\left(P_{c}\left(1-x^{2}\right)\right)^{n-1}+1\right)+1\right)^{-1},
\end{aligned}
$$

and, $P_{c}$, probability of transmitted packet encounter collision when $N$ is number of whole nodes, is given by

$$
P_{c}=1-(1-\tau)^{N-1},
$$

also, $K_{0}=L_{0} q_{0} /\left(1-q_{0}\right)$ whereas, $L_{0}$ is the idle state length without generating packets and, $q_{0}$ is the probability of going back to the idle state. Consider

$$
\begin{aligned}
& x=\alpha+(1-\alpha) \beta, \\
& y=P_{c}\left(1-x^{m+1}\right) .
\end{aligned}
$$

The busy channel probabilities (CCA1) and (CCA2) are $\alpha, \beta$, respectively, given as follows:

$$
\alpha=\alpha_{1}+\alpha_{2}
$$

where

$$
\begin{gathered}
\alpha_{1}=L\left(1-(1-\tau)^{N-1}\right)(1-\alpha)(1-\beta), \\
\alpha_{2}=L_{\text {ack }} \frac{N \tau(1-\tau)^{N-1}}{1-(1-\tau)^{N}}\left(1-(1-\tau)^{N-1}\right)(1-\alpha)(1-\beta),
\end{gathered}
$$


with

$$
\beta=\frac{1-(1-\tau)^{N-1}+N \tau(1-\tau)^{N-1}}{2-(1-\tau)^{N}+N \tau(1-\tau)^{N-1}} .
$$

While the Markov chain just declared the probability of collision $P_{c}$ as cause of loss, we bring $P_{c s i}$ which is derived in (18), into the account as another possibility of loss due to different SNR, modulation, and channel coding. Probability of failure is defined as

$$
P_{\text {fail }}=1-\left(1-P_{c}\right)\left(1-P_{\text {csi }}\right),
$$

where $P_{c}$ is given in (23) as probability of packet collision. Cluster network could be modeled with binominal random variable with independent $j$ th clusters $D_{c}^{(j)}$, where $j=$ $1, \ldots, n_{c}, d_{c}^{(j)}$ is referring to cluster size, denotes a probability $p_{\text {mac }}\left(d_{c}^{(j)}\right)$ corresponding to $j$ th cluster. Performance metric expression that has been extracted from Markov model could be updated according to our assumptions:

$$
P(\mathfrak{J})=\sum_{i_{1}=0}^{d_{c}^{(1)}} \cdots \sum_{i_{n_{c}}=0}^{d_{c}^{\left(n_{c}\right)}} \mathbb{P}\left\{D^{(1)}=i_{1}\right\} \cdots \mathbb{P}\left\{D^{\left(n_{c}\right)}=i_{n_{c}}\right\},
$$

where $\mathfrak{I}$ denotes possible variable which could be computed by

$$
\mathbb{P}\left\{D^{(\ell)}=i_{\ell}\right\}=\left(\begin{array}{c}
d_{c}^{(\ell)} \\
i_{\ell}
\end{array}\right)\left[p_{\mathrm{mac}}\left(d_{c}^{(\ell)}\right)\right]^{i_{\ell}}\left[1-p_{\mathrm{mac}}\left(d_{c}^{(\ell)}\right)\right]^{d_{c}^{(\ell)}-i_{\ell}}
$$

Using Markov chain performance metric equations, we will be obtaining the following.

3.5.1. Reliability. The probability of successful delivery of packets $\mathbf{R}$ as a clustering topology network, regarding reliability in [2], (31), and (30) redefining the probability of successful delivery of packets majority sensors per cluster which satisfy majority-like fusion strategy, is:

$$
P_{R}^{i_{\ell}}=\sum_{i_{\ell}=\chi}^{d_{c}^{(\ell)}} \prod_{\ell=1}^{n_{c}}\left(\begin{array}{c}
d_{c}^{(\ell)} \\
i_{\ell}
\end{array}\right)\left[\mathbf{R}\left(d_{c}^{(\ell)}\right)\right]^{i_{\ell}}\left[1-\mathbf{R}\left(d_{c}^{(\ell)}\right)\right]^{d_{c}^{(\ell)}-i_{\ell}}
$$

where $\chi=\left\lfloor d_{c}^{(\ell)} / 2\right\rfloor+1, \ell=\left\{1, \ldots, n_{c}\right\}$. Two-level fusion at FC and AP, $P_{R}^{i_{\ell}}$ is given as the probability of successful delivery distributed sensors in first level fusion; the probability of successful delivery FC to AP has similarity by assuming as a cluster with $n_{c}$ sensors for second level fusion. Hence, reliability equation for both levels of fusion at $\mathrm{FC}$ and $\mathrm{AP}$ is remarked with $\mathbf{R}_{c}$ given by,

$$
\mathbf{R}_{c}=P_{R}^{i_{e}} \cdot P_{R}^{(f c)},
$$

where $P_{R}^{(f c)}$ is obtained from (30) and (31) with $d_{c}^{(f c)}=n_{c}$ for second level.
3.5.2. Average Delay. It is noted that communication delays can deteriorate the performance of the network and even can destabilize the systems when they are not considered in the design (see $[20,21])$. Therefore, the average delay for clustering with two-level fusion is defined as average delay of successfully received packet as the time interval from the instant the packet is at the head of its MAC queue and ready to be transmitted, until the transmission is successful and the ACK is received from both level of fusion nodes, respectively. According to [2], in framework except the constants (frame length, Ack length, etc.), MAC parameters have only two terms, $\operatorname{Pr}\left(A_{j} \mid A_{t}\right)$ and $\widetilde{P}\left(B_{i} \mid B_{t}\right)$ that could be computed based on (30) and (31). However, initially $\alpha, \beta, \tau$ should be calculated with respect to a given topology at clusters and also $x, y$, and $P_{c}$ with term (29). Obviously, MAC parameters are similar for all equations with optimal tune. Framework concerning majority-like mechanism should be taken into account when encountered with $N$ number sensors in original Markov chain equations that are replaced by $\left\lfloor d_{c} / 2\right\rfloor+$ $1, \ldots, d_{c}$ for each cluster by corresponding sensors. So far, average delay is described for first level fusion of each cluster separately. For second fusion level, it is acting as a cluster with $n_{c}$ sensors. Average delay of whole network is proposed by:

$$
\mathbb{E}_{c}^{(\text {av })}[\widetilde{D}]=\frac{\mathbb{E}_{\max }+\mathbb{E}_{\min }}{2}+\mathbb{E}^{(f c)}[\widetilde{D}],
$$

where, $\mathbb{E}_{\max }=\operatorname{Max}\left\{\mathbb{E}_{c}^{(1)}[\widetilde{D}], \ldots, \mathbb{E}_{c}^{\left(n_{c}\right)}[\widetilde{D}]\right\}$ and $\mathbb{E}_{\min }=$ $\operatorname{Min}\left\{\mathbb{E}_{c}^{(1)}[\widetilde{D}], \ldots, \mathbb{E}_{c}^{\left(n_{c}\right)}[\widetilde{D}]\right\}$, first term is average delay for which packets arriving for first level fusion at FC clusters head, and second term for which packets arriving second level fusion at AP. Because of synchronized network, transmission happens at the same time and concurrently; hence, Max and Min are computed regarding to cluster size and parameters.

3.5.3. Network Aggregate Throughput. Network aggregate throughput would be computed for minimum effective number of nodes each cluster network with two-level fusion and data rate $g$ (bps) is given by:

$$
S_{c}=g \cdot A \cdot L_{s} \cdot \hbar \cdot \mathbf{R}_{c},
$$

where $\hbar=\sum_{\ell=1}^{n_{c}}\left\lfloor d_{c}^{(\ell)} / 2\right\rfloor+1, \mathbf{R}_{c}$ is computed at (33) and $A=$ $80 \mathrm{bit} / 0.32 \mathrm{~ms}$ is a normalization constant to convert to bps.

3.5.4. Average Power Consumption. The average power consumption equations are proposed in $[2,14]$ taken into consideration by the clustering framework with two-level fusion. Constant values given in Table 2 are used for first level fusion; however, for second level fusion they are valid except $P_{i} \approx 0$ because of assuming fusion center does not have ideal state at second level, also hypothesis $P_{\text {sc }}$ sensing power constant at sensor is corresponding with power of decision-making at fusion node and assumed same computation term.

\section{Simulation Results}

This section represents the results of simulation based on problem framework. Basically, simulations are figured out 
TABle 2: Power consumption of different operation modes.

\begin{tabular}{lc}
\hline Operation mode & Power consumption \\
\hline$P_{i}$ & $0.657 \mathrm{~mW}$ \\
$P_{\mathrm{sc}}$ & $35.46 \mathrm{~mW}$ \\
$P_{t}$ & $31.32 \mathrm{~mW}$ \\
$P_{r}$ & $35.46 \mathrm{~mW}$ \\
\hline
\end{tabular}

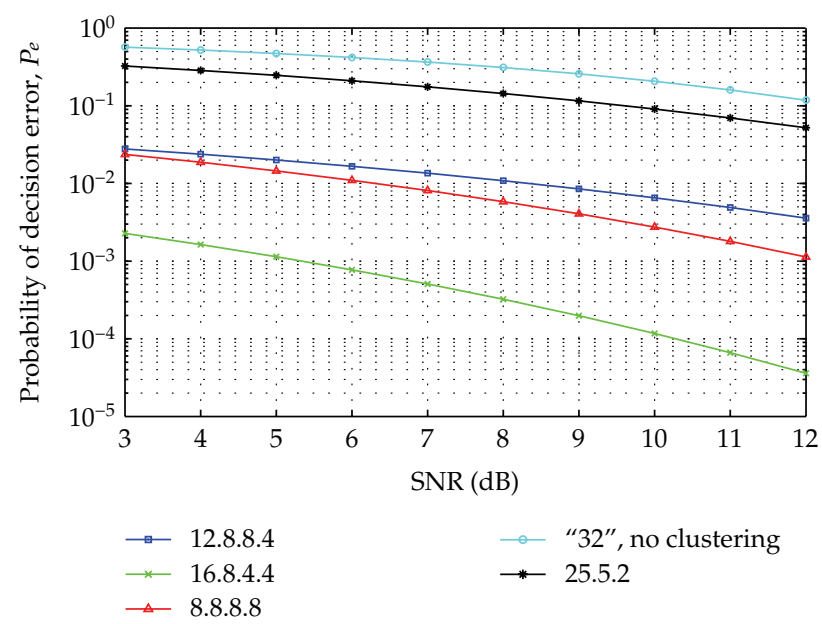

FIgURE 3: Probability of decision error as a function of SNR, $n=32$ sensors with AWGN.

with " 32 " nodes as detector of an event of interest, each node generates high traffic data rates. Performance metric is evaluated with probability of decision error and developed equations of Markov model. Rest of section is organized into two subsections based on those evaluations.

4.1. Probability of Decision Error. Simulation results shows for evaluating probability of decision error in fusion center based on described framework. Probability of decision error is considered at fusion node with respect to clustering topology as long as presence of uniform and non-uniform distributions of " 32 " sensors. Three non-uniform distributions 12.8.8.4, 16.8.4.4, and 25.5.2 are versus uniform distribution 8.8.8.8. Non-clustering by " 32 " sensors are shown as a proof of comparison in Figure 3. It represents the probability of decision error for non-clustering topology which looks like a star network with coordinator acting as fusion node. Detection sequences just effect with Additive White Gaussian Noise (AWGN) communication channel with OQPSK modulation format.

Basically, an increment of SNR has improvement on decision. According to various sensors distribution, Figure 3 is shown that non-clustering is worst case with respect to our scenario; the decision is made at fusion based on vector received signals on majority-like strategy. Hence, in case of non-clustering at least 17 sensors similar to record as correct decision should be received but for clustering this limitation reduces to $\left[d_{c} / 2\right]+1$. Number of sensors at each cluster for example in 16.8.4.4 design by 4 clusters have 16, 8, 4 , 4 sensors at each cluster; therefore, fusion node at head of clusters

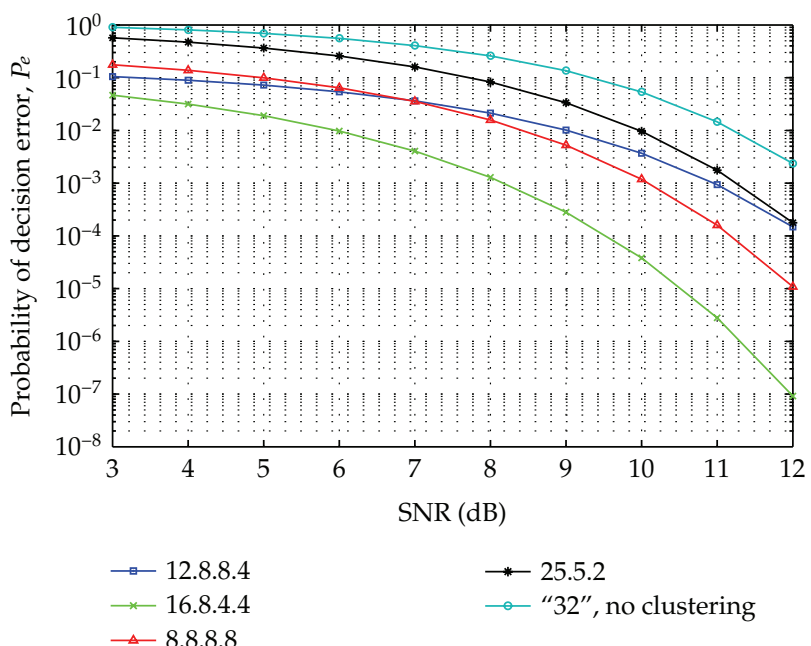

Figure 4: Probability of decision error versus SNR, $n=32$ sensors with $P_{\mathrm{csi}}$.

TABle 3: Parameters value for physical layer.

\begin{tabular}{lc}
\hline Parameter & Value \\
\hline Minimum distance & 1 meter \\
Maximum distance & 40 meters \\
Frame length & $808 \mathrm{bits}$ \\
Power Tx & $3 \mathrm{~dB} \mathrm{~m}$ \\
pramble length & $40 \mathrm{bits}$ \\
Noise figure & $-123 \mathrm{~dB} \mathrm{~m}$ \\
Noise & $-5 \mathrm{~dB} \mathrm{~m}$ \\
Band width & $30 \mathrm{kHz}$ \\
Signal frequency $(f)$ & $2450 \mathrm{MHz}$ \\
Path loss exponent & 4 \\
Shadowing standard deviation & 4 \\
\hline
\end{tabular}

should be evaluated 9, 5, 3, 3 signals similarly which have same level for corresponding clusters. However, in second level decision-making at AP should be outcome of decision on first level satisfies with 3 similar signals received form 4 fusion nodes.

Figure 4 shows the probability of decision error with presence of $P_{c s i}$ and fading effect. Impact of $P_{c s i}$ and fading effect are measured by attenuation on level of signal to change probability in order to increscent decision error due to channel influence. According to literatures of Monte Carlo simulation of corresponding expressions in given framework has confirmed our simulation. MAC and PHY parameters values used for $P_{\text {csi }}$ are shown in Table 3.

4.2. Performance Metric Evaluation Based on Markov Chain. Impact of MAC appraised on proposed framework. Simulation of performance metric equations is carried out with MAC and PHY-layers parameters denoted in Tables 3 and 4.

4.2.1. Reliability. Reliability is obtained for non-uniform and uniform topology which is supposed to be with different 
TABLE 4: Parameters value for MAC layer.

\begin{tabular}{lc}
\hline Parameter & Value \\
\hline MacMaxFrameRetries $(n)$ & 3 \\
MacMaxCSMABackoffs $(m)$ & 4 \\
MacMinBE $\left(m_{0}\right)$ & 3 \\
MacMaxBE $\left(m_{b}\right)$ & 5 \\
$L$ & 1016 bits \\
$L_{\text {ack }}$ & 88 bits \\
$t_{\text {ack }}$ & $222 e-9$ seconds \\
$t_{\text {IFS }}$ & $640 e-6$ seconds \\
$t_{m \text {,ack }}$ & $200 e-9$ seconds \\
aUnitBackoffPeriod & $320 e-6$ seconds \\
macACKWaitDuration & $1920 e-6$ seconds \\
aTurnaroundTime & $192 e-6$ seconds \\
$S_{b}$ & $128 e-6$ seconds \\
$L_{0}$ & $10 e-12$ \\
$W_{0}$ & $2^{\text {macMinBE }}$ \\
$q_{0}$ & $10 e-12$ \\
\hline
\end{tabular}

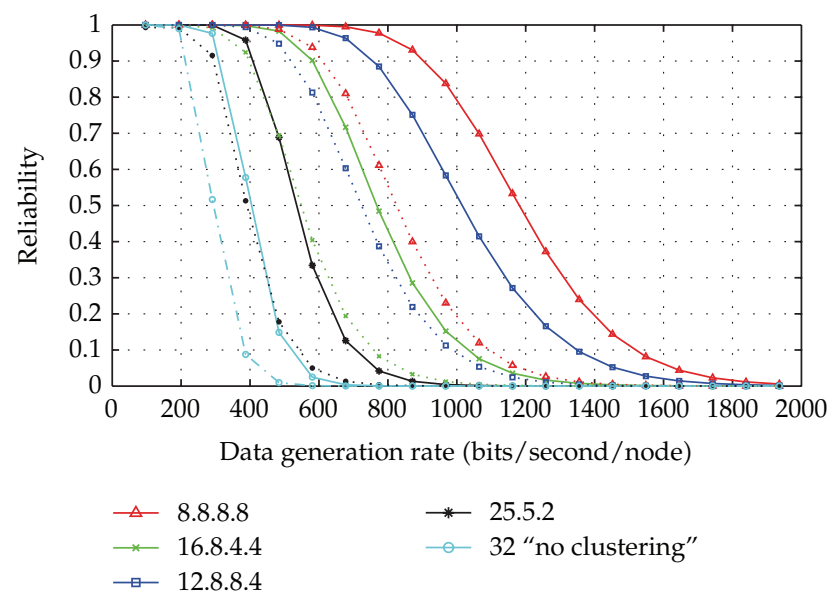

Figure 5: Reliability, $E_{b} / N_{0}=3,12[\mathrm{~dB}]$, OQPSK.

number of sensors at each clusters. Model is evaluated in high data rate generation. Three non-uniformly distribution 16.8.4.4, 25.5.2, and 12.8.8.4 at each cluster and uniformly 8.8.8.8 distribution sensors are compared by non-clustering which is similar to a star topology that originally was assumed in Markov chain model. Result shows a significant improvement in reliability in clustering topology even in two-level fusion. However, in clustering based topologies balance of sensors distribution (uniform) in clusters are more reliable than unbalances (non-uniform). Figure 5 represents the reliability of system with signal to noise ratio equal to $12 \mathrm{~dB}$ in solid line by comparison with $3 \mathrm{~dB}$ in dots line. Reliability is enhanced in order to increment signal to noise ratio. Direct relation between probability of success packet reception or reliability, with probability of packet failure shown in Figure 6, has consequent improvement on reliability. Increasing SNR from $3 \mathrm{~dB}$ to $12 \mathrm{~dB}$ causes less failure packet reception at fusion node. Result represents in

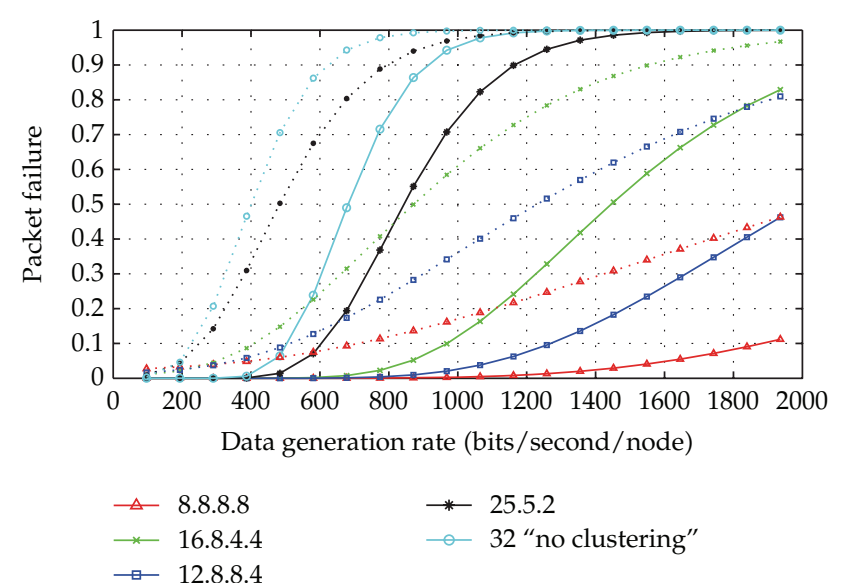

FIgURE 6: Failure probability, $E_{b} / N_{0}=3,12$ [dB], OQPSK.

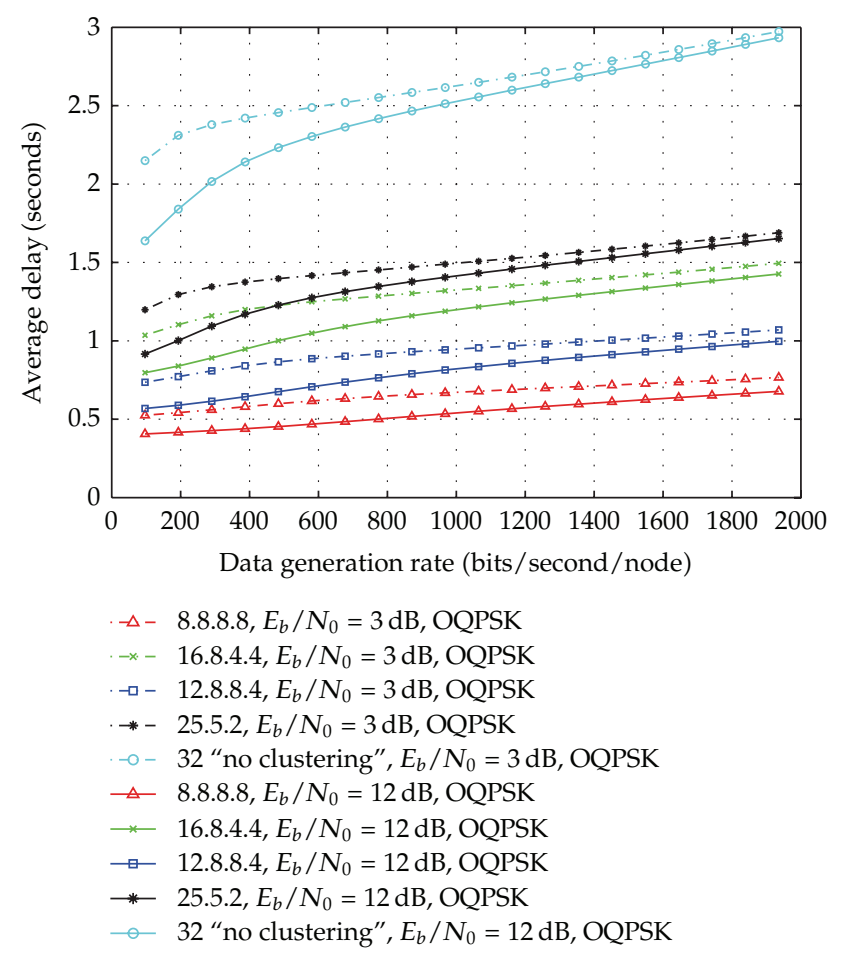

FIgURE 7: Average delay for two-level decision.

probability of decision error $P_{e}$ also proving this improvement at FC. However, packet failure is increased versus packet generation rate; therefore, we can expect high packet generation rate more effective than increment of SNR ratio in failure term.

4.2.2. Average Delay. Measurement of average delay is explained in framework. Simulation performs with high traffic regime with two SNRs $3 \mathrm{~dB}$ and $12 \mathrm{~dB}$, see Figure 7 . Important issue here is synchronizing between nodes by specifying a time slot from FC to nodes for retrieve data. Obviously, this time slot is corresponding to size of each cluster, therefor, time slot for cluster with 8 sensors is four 


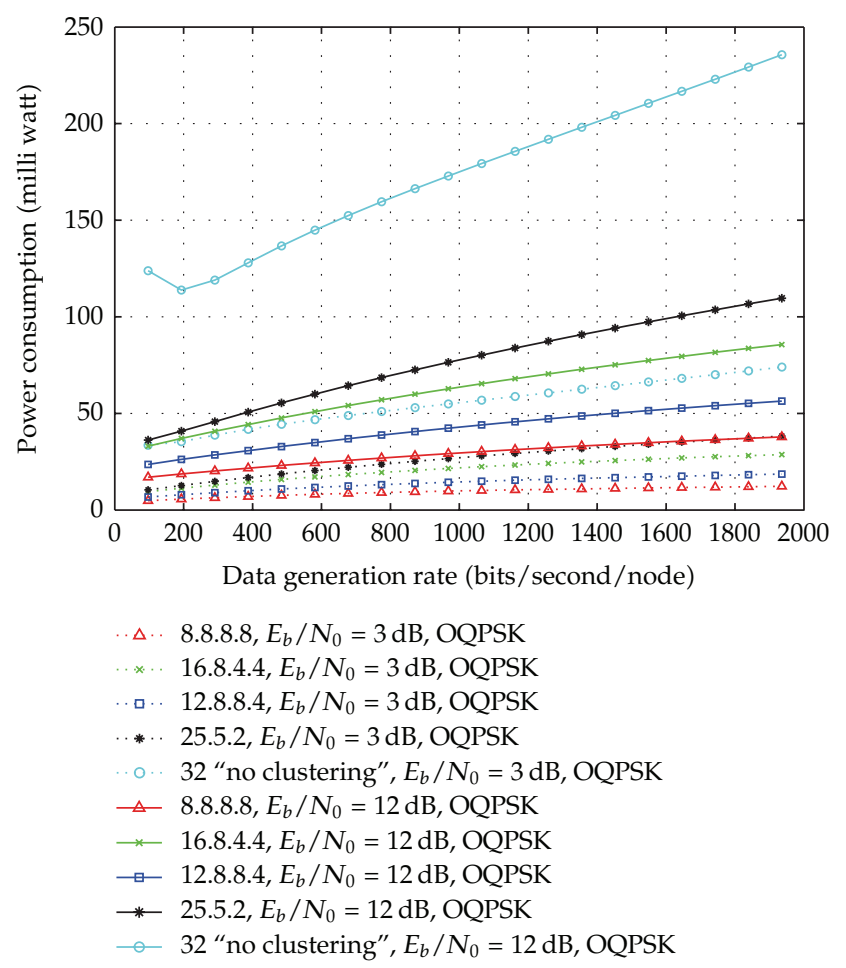

Figure 8: Average power consumption for two-level decision.

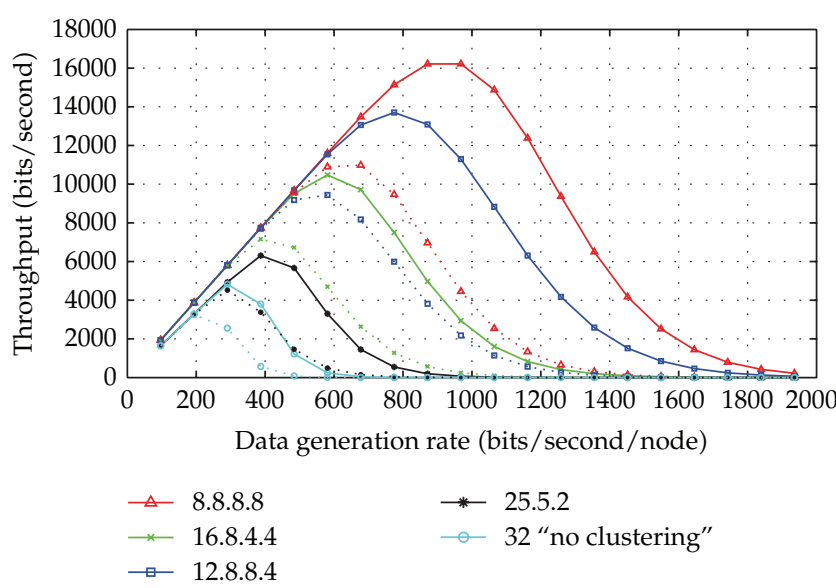

FIgURE 9: Throughput, $E_{b} / N_{0}=3,12[\mathrm{~dB}]$, OQPSK.

times greater than time slot for cluster with 2 sensors because of preventing collision in each cluster during transmitting and each node of cluster has its own time slot to send. Clusters are independent from each other and transmit in their appropriated bandwidth. IEEE 802.15 .4 has 16 channels in $2.4 \mathrm{GHz}$, based on simulation with maximum 4 clusters there is not any constraint in bandwidth scheduling; hence, each cluster works in a unique bandwidth. Slotted Markov chain model specification satisfies the condition. Non-clustering topology has more average delay. That delay is imaginable because time slot scheduling scenario for " 32 " nodes need longer time slot length. Uniformed distribution 8.8.8.8 needs a time slot with 8 portions at each cluster for retrieving data process. Clusters that have more nodes need at least a time slot longer than with 8 portions. Basically, effect of number sensors on $\alpha$ and $\beta$ and $\tau$ are important exact contribution of less sensors causes increasing probability of access channel and directly reduces delays.

4.2.3. Average Power Consumption. An increment of mean power consumption with higher data generation rate obviously is illustrated in Figure 8. Basically, data transmission consumes more power rather than computational matter in sensor module. Nevertheless, number of sensors and fusion level have critical roles to achieve power consumption. Topology without clustering " 32 " sensors contribute in decision-making in fusion node coordinates at least half plus one received bits stream signal. Average power is increased with $12 \mathrm{~dB}$ signal to noise ratio due to transmission power consumption, $P_{s}$. While power consumption is a very critical issue to wireless sensor network, increment of power consumption is unwilling with respect to restriction on battery capability. On the other view, preciseness of packet receipt sometimes has privilege to power consumption.

4.2.4. Network Aggregate Throughput. Network aggregate throughput is shown in Figure 9 as function of data generation rate with two SNR ratios. Throughput relation with reliability is explained in framework description. All issues represent improvement in higher signal to noise ratio. Throughput reduction happens when data generation rate is up to 900 bits per seconds in each node.

\section{Conclusion}

This paper considered a distributed detection in cluster sensor network with fusion node as a decision maker head of each cluster. We utilized a Markov chain model for evaluation network performance. Generally speaking, network clusterbased topology with data fusion has better performance with aim of data accuracy. Presence of clustering with balance distribution of sensors is acting more efficiently than nonuniform clustering with more number of distributed sensors. Number of distribution sensors directly impacts average delay in clusters; hence, a topology should be selected for less delay achievement. Throughput has better outcome in cluster-based with balance distribution sensors. Power consumption has been acting better in uniformly distributed topology instead of non-uniformly as well as clustering and non-clustering. The main reason of this difference between sensor arrangements is scheduling and timing issue on network. Those issues influence directly on average delay and power consumption. However, it can affect packet failure and also reliability of system.

Based on the results in the paper, interesting future research may be prospective as follows:

(1) optimized sensor arrangement in cluster and network state estimation could be considered;

(2) fault detection and time delays in the network with Markovian jump systems under partially known 
transition probabilities can be studied in the framework of this paper (see for instance [22-25]);

(3) the approach, presented in this work, can also be extended to complex networks with constrained information exchange, and a partial knowledge of the state variables (see $[26,27])$.

\section{References}

[1] IEEE Std 802.15.4-2996, September, Part 15.4: Wireless Medium Access Control(MAC) and Physical Layer (PHY) Specifications for Low-Rate Wireless Personal Area Networks (WPANs), IEEE, 2006, http://www.ieee802.org/15.

[2] P. Park, P. Di Marco, P. Soldati, C. Fischione, and K. H. Johansson, "A generalized Markov chain model for effective analysis of slotted IEEE 802.15.4," in Proceedings of IEEE 6th International Conference on Mobile Adhoc and Sensor Systems (MASS '09), pp. 130-139, October 2009.

[3] M.-H. Zayani, V. Gauthier, and D. Zeghlache, "A joint model for IEEE 802.15.4 physical and medium access control layers," in Proceedings of the 7th International Wireless Communications and Mobile Computing Conference (IWCMC '11), Istanbul, Turkey, 2011.

[4] G. Zhou, Z. Zhu, G. Chen, and L. Zhou, "Decision fusion rules based on multi-bit knowledge of local sensors in wireless sensor networks," Information Fusion, vol. 12, no. 3, pp. 187-193, 2011.

[5] G. Ferrari, P. Medagliani, M. Martalò, and A. Muzzini, "Zigbee sensor networks with data fusion," in Proceedings of the $3 \mathrm{rd}$ International Symposium on Communications, Control, and Signal Processing (ISCCSP '08), pp. 472-477, March 2008.

[6] G. Ferrari, M. Martalò, and R. Pagliari, "Decentralized detection in clustered sensor networks," IEEE Transactions on Aerospace and Electronic Systems, vol. 47, no. 2, pp. 959-973, 2011.

[7] M. Martalò, C. Buratti, G. Ferrari, and R. Verdone, "Decentralized detection in IEEE 802.15.4 wireless sensor networks," Eurasip Journal on Wireless Communications and Networking, vol. 2010, article 3, Article ID 174063, 10 pages, 2010.

[8] M. Martalò and G. Ferrari, "A simple information-theoretic analysis of clustered sensor networks with decentralized detection," IEEE Communications Letters, vol. 14, no. 6, pp. 560-562, 2010.

[9] M. Martal and G. Ferrari, "Decoding and fusion in distributed detection schemes with unreliable communications," IEEE Transactions on Aerospace and Electronic Systems, vol. 48, no. 1, pp. 16-26, 2012.

[10] G. Ferrari, P. Medagliani, S. Di Piazza, and M. Martalò, "Wireless sensor networks: performance analysis in indoor scenarios," Eurasip Journal on Wireless Communications and Networking, vol. 2007, Article ID 81864, 2007.

[11] H. Chen, B. Chen, and P. K. Varshney, "A new framework for distributed detection with conditionally dependent observations," IEEE Transactions on Signal Processing, vol. 60, no. 3, pp. 1409-1419, 2012.

[12] M. Z. Zamalloa and B. Krishnamachari, "An analysis of unreliability and asymmetry in low-power wireless links," $A C M$ Transactions on Sensor Networks, vol. 3, no. 2, Article ID 1240227, 2007.

[13] P. Park, P. Di Marco, C. Fischione, and K. H. Johansson, Adaptive IEEE 802.15.4 Protocol for Reliable and Timely Communications, KTH, 2009.
[14] P. Park, C. Fischione, and K. H. Johansson, "Adaptive IEEE 802.15.4 protocol for energy efficient, reliable and timely communications," in Proceedings of the 9th ACM/IEEE International Conference on Information Processing in Sensor Networks (IPSN '10), pp. 327-338, ACM, April 2010.

[15] S. Jin, W. Yue, and Q. Sun, "Performance analysis of the sleep/wakeup protocol in a wireless sensor network," International Journal of Innovative Computing Information and Control, vol. 8, no. 5, pp. 3833-3844, 2012.

[16] M. I. Razzak, B. A. Elmogy, M. K. Khan, and K. Alghathbar, "Efficient distributed face recognition in wireless sensor network," International Journal of Innovative Computing, Information and Control, vol. 8, no. 4, pp. 2811-2822, 2012.

[17] R. Verdone, D. Dardari, G. Mazzini, and A. Conti, Wireless Sensor and Actuator Networks, Technology, Analysis, Design, chapter 9, Academic Press, 2007.

[18] C. Buratti, "Performance analysis of IEEE 802.15 .4 beaconenabled mode," IEEE Transactions on Vehicular Technology, vol. 59, no. 4, pp. 2031-2045, 2010.

[19] F. Kojima and J. S. Knopp, "Inverse problem for electromagnetic propagation in a dielectric medium using Markov chain Monte Carlo method," International Journal of Innovative Computing Information and Control, vol. 8, no. 3, pp. 2339-2346, 2012.

[20] H. R. Karimi, "Robust $H_{\infty}$ filter design for uncertain linear systems over network with network-induced delays and output quantization," Modeling, Identification and Control, vol. 30, no. 1, pp. 27-37, 2009.

[21] H. R. Karimi and H. Gao, "New delay-dependent exponential $H_{\infty}$ synchronization for uncertain neural networks with mixed time delays," IEEE Transactions on Systems, Man, and Cybernetics B, vol. 40, no. 1, pp. 173-185, 2010.

[22] X. Su, L. Wu, and P. Shi, "Senor networks with random link failures: distributed filtering for T-S fuzzy systems," IEEE Transactions on Industrial Informatics. In press.

[23] L. Wu, X. Su, and P. Shi, "Sliding mode control with bounded $L_{2}$ gain performance of Markovian jump singular time-delay systems," Automatica, vol. 48, no. 8, pp. 1929-1933, 2012.

[24] H. R. Karimi, "Robust delay-dependent $H_{\infty}$ control of uncertain time-delay systems with mixed neutral, discrete, and distributed time-delays and Markovian switching parameters," IEEE Transactions on Circuits and Systems. I. Regular Papers, vol. 58, no. 8, pp. 1910-1923, 2011.

[25] L. Wu, X. Yao, and W. X. Zheng, "Generalized $H_{2}$ fault detection for two-dimensional Markovian jump systems," Automatica, vol. 48, no. 8, pp. 1741-1750, 2012.

[26] R. Yang, P. Shi, G.-P. Liu, and H. Gao, "Network-based feedback control for systems with mixed delays based on quantization and dropout compensation," Automatica, vol. 47, no. 12, pp. 2805-2809, 2011.

[27] L. Wu, P. Shi, and H. Gao, "State estimation and slidingmode control of Markovian jump singular systems," IEEE Transactions on Automatic Control, vol. 55, no. 5, pp. 1213-1219, 2010. 


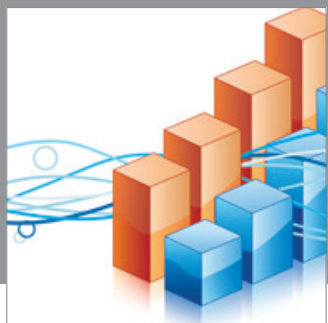

Advances in

Operations Research

mansans

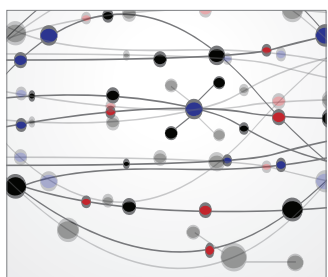

The Scientific World Journal
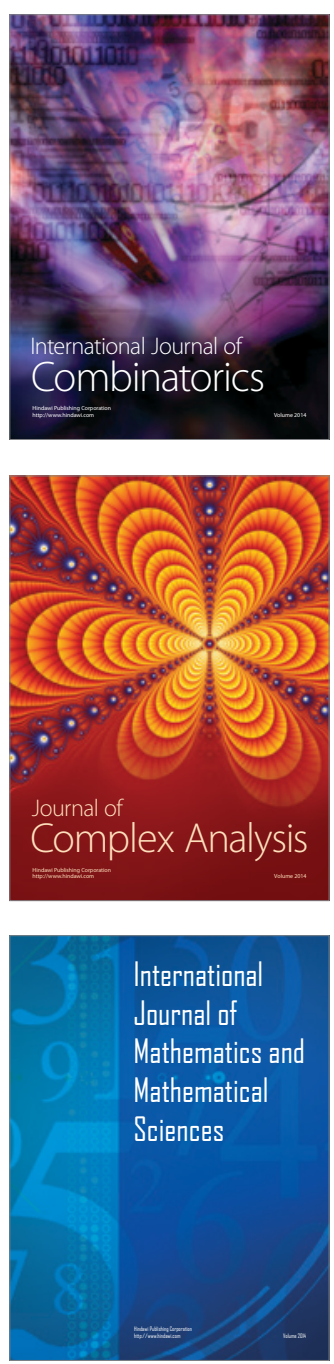
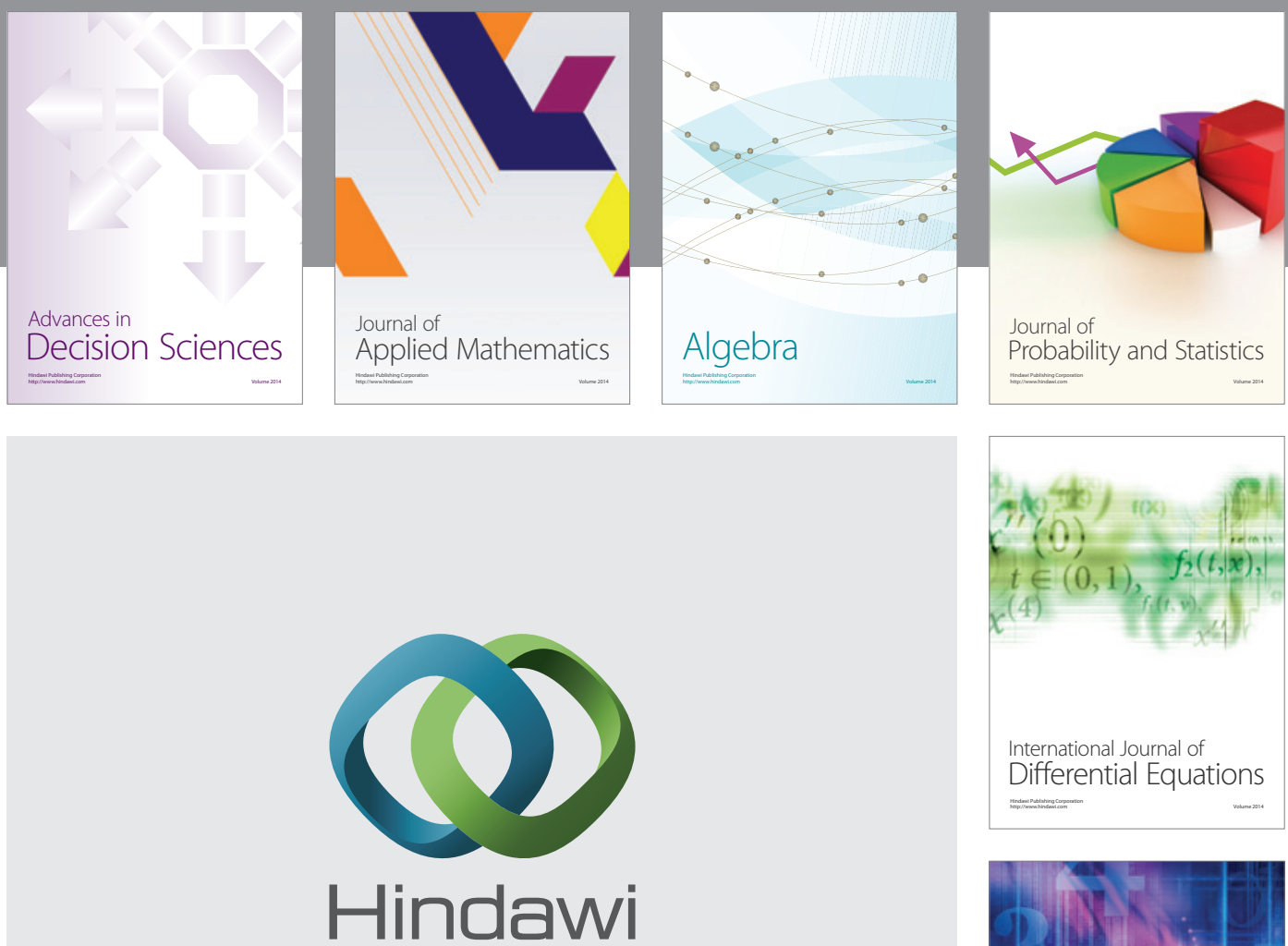

Submit your manuscripts at http://www.hindawi.com
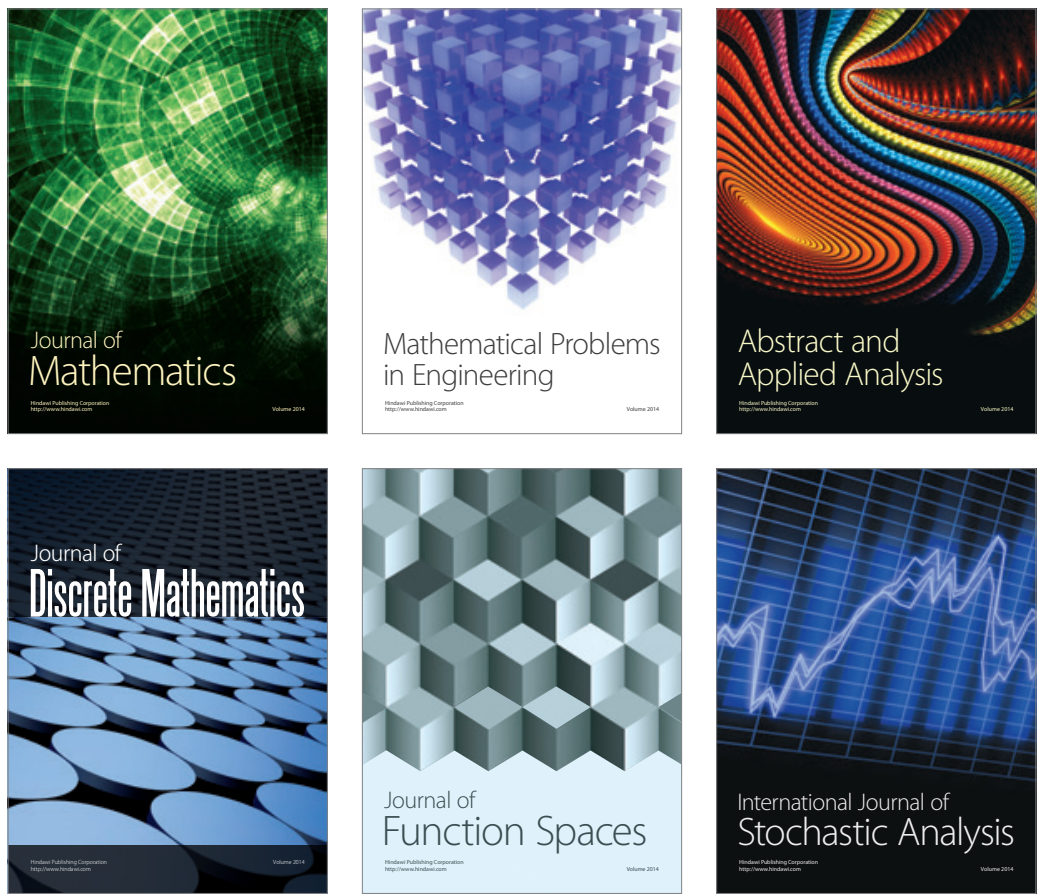

Journal of

Function Spaces

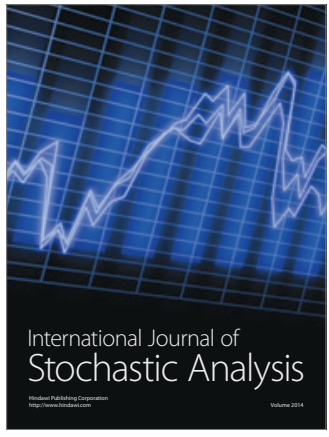

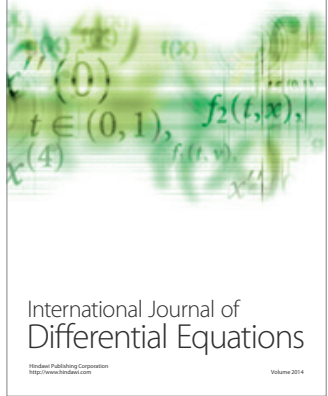
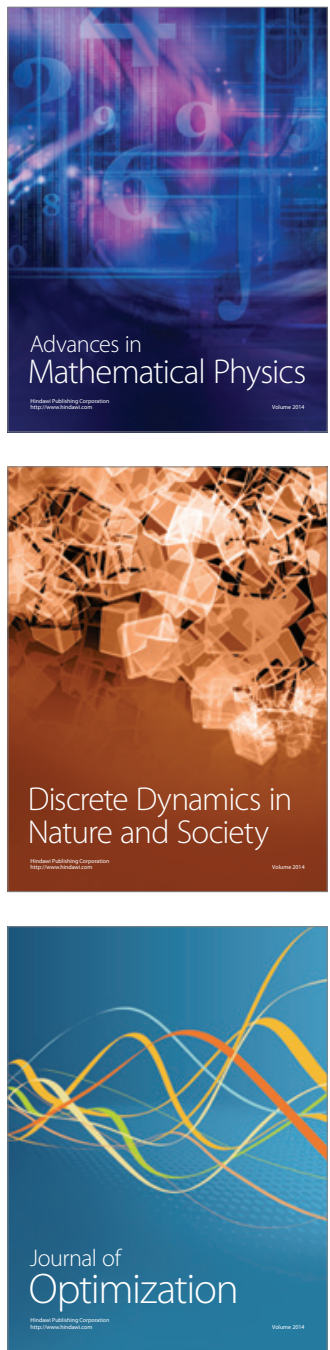